UCRL-ID-119958

\title{
Rationale and Summary of Methods for Determining Ultrasonic Properties of Materials at Lawrence Livermore National Laboratory
}

Albert E. Brown

February 9,1995

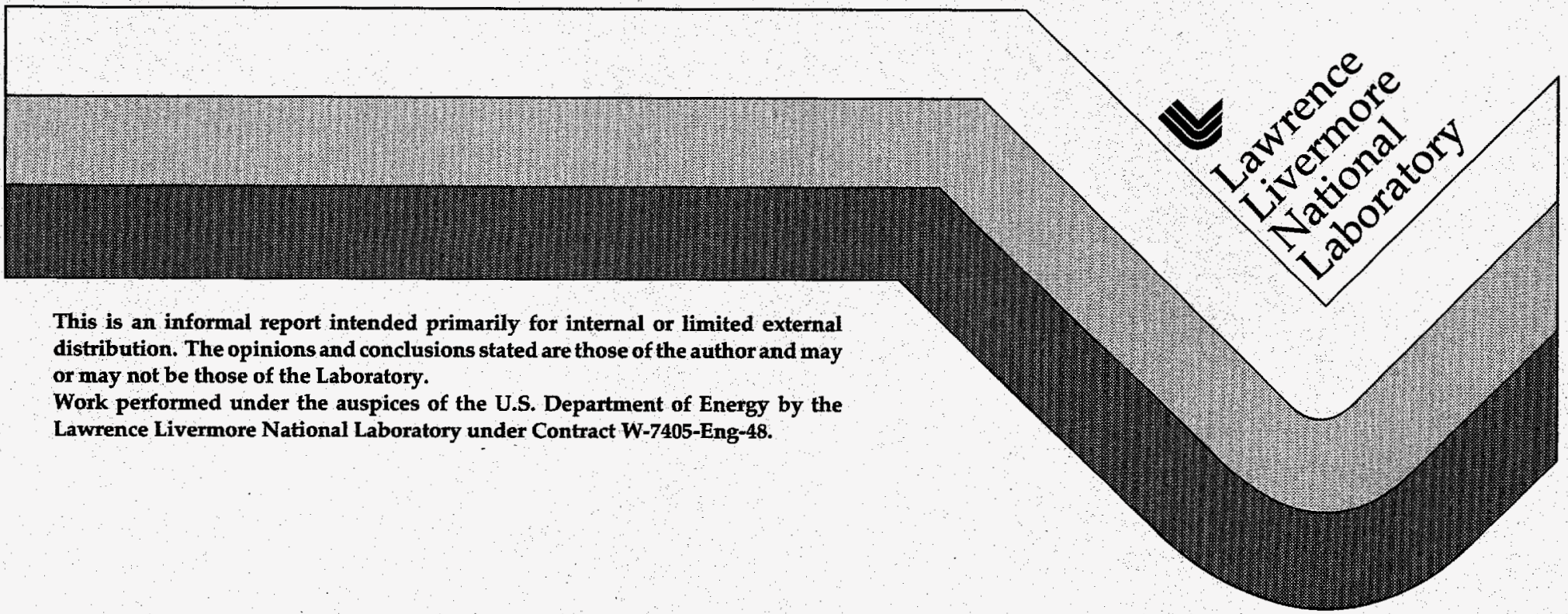




\section{DISCLAIMER}

This document was prepared as an account of work sponsored by an agency of the United States Government. Neither the United States Government nor the University of California nor any of their employees, makes any warranty, express or implied, or assumes any legal liability or responsibility for the accuracy, completeness, or usefulness of any information, apparatus, product, or process disclosed, or represents that its use would not infringe privately owned rights. Reference herein to any specific commercial product, process, or service by trade name, trademark, manufacturer, or otherwise, does not necessarily constitute or imply its endorsement, recommendation, or favoring by the United States Government or the University of California. The views and opinions of authors expressed herein do not necessarily state or reflect those of the United States Government or the University of California, and shall not be used for advertising or product endorsement purposes.

This report has been reproduced directly from the best available copy.

Available to DOE and DOE contractors from the Office of Scientific and Technical Information P.O. Box 62, Oak Ridge, TN 37831

Prices available from (615) 576-8401, FTS 626-8401

Available to the public from the National Technical Information Service

U.S. Department of Commerce 5285 Port Royal Rd.

Springfield, VA 22161 


\section{DISCLAIMER}

Portions of this document may be illegible in electronic image products. Images are produced from the best available original document. 


\section{RATIONALE AND SUMMARY OF METHODS FOR DETERMINING ULTRASONIC PROPERTIES OF MATERIALS AT LAWRENCE LIVERMORE NATIONAL LABORATORY}

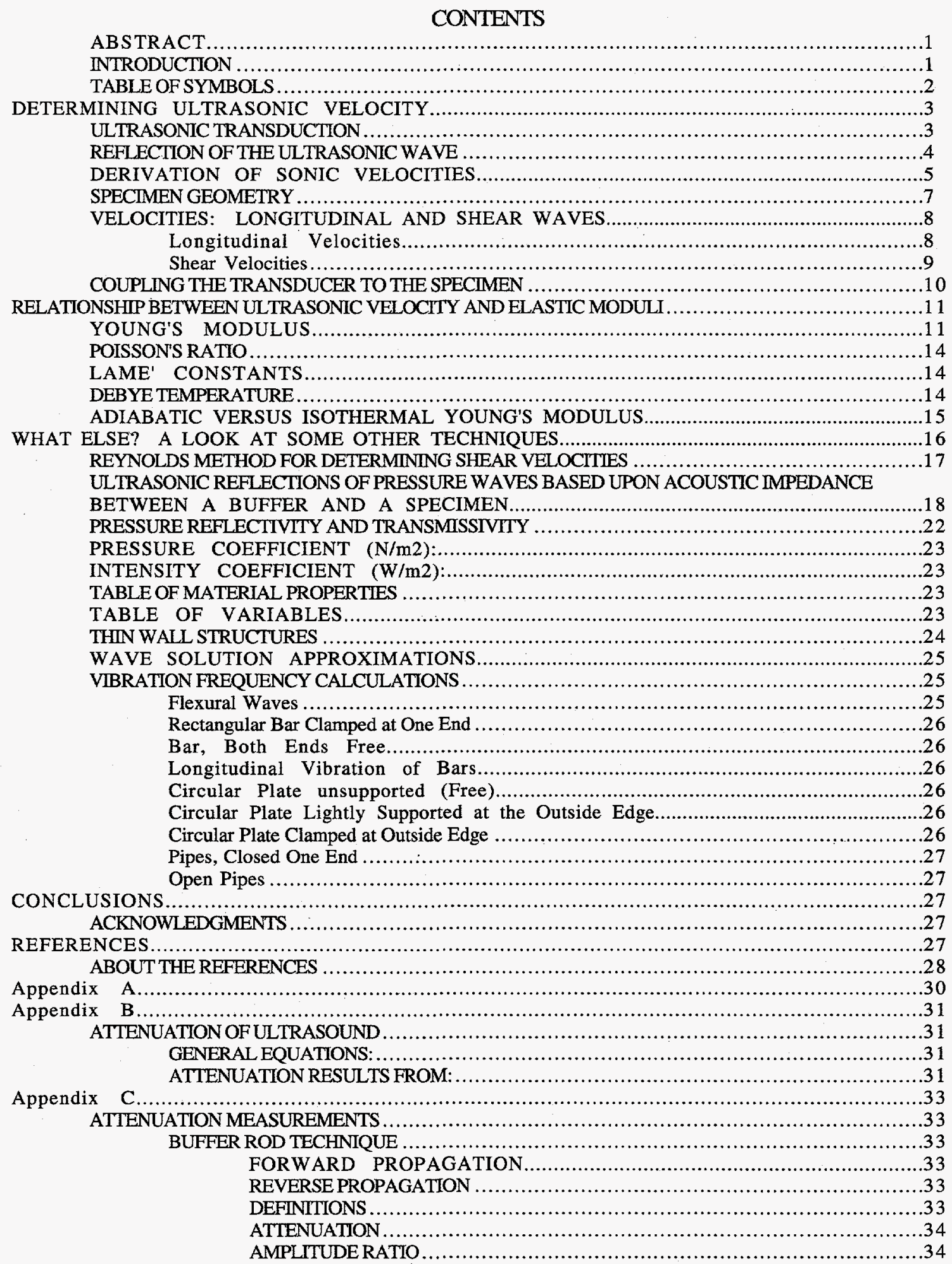




\title{
RATIONALE AND SUMMARY OF METHODS FOR DETERMINING ULTRASONIC PROPERTIES OF MATERIALS AT LAWRENCE LIVERMORE NATIONAL LABORATORY*
}

\begin{abstract}
This report is a summary of the methods used to determine ultrasonic velocities through the many materials tested at the Acoustic Properties of Materials Laboratory. Ultrasonic velocity techniques enable us to determine material properties, including elastic moduli, without harming the materials being tested, an advantage some over mechanical methods. Ultrasonic modulus determination has other advantages as well: 1.) relative ease and low cost of material preparation, and, 2.) comparative analysis to physical testing as a function of material loading rate dependence. In addition, ultrasonic measurement provides clues to determine grain size and orientation, and provides a relative indication of material anisotropy with respect to the material geometry. We usually perform ultrasonic measurements on materials in ambient atmospheric conditions, and in a relatively free-free condition. However, we can perform them in other environments, as required.
\end{abstract}

This paper describes some of our techniques and shows how ultrasonic velocities are used to establish elastic constants. It also includes a sample test report for a homogeneous isotropic solid, along with a list of references.

\section{INTRODUCTION}

Questions are frequently asked about the techniques used at the Lawrence Livermore National Laboratory to determine the velocity of sound through the various materials examined in the Acoustic Properties of Materials Laboratory, Manufacturing \& Materials Engineering Division. Acoustic techniques enable us to measure material properties without harming the materials being tested, an important advantage over other methods. This paper describes some of the techniques and foibles used to measure sonic velocities and how these velocities are used to establish the elastic constants of investigated materials. However, it is not intended, nor should the reader infer that this report is comprehensive, since there is a limitation on how much a report of this size should contain. Moreover, there are a great many books available for the reader who wishes more detail. Books on elastic waves generally fall into two broad categories: applications and theory. The 
bibliography at the end of this report lists selected material in these two areas of elastic wave research. An example of a test report is given in Appendix A'.

TABLE OF SYMBOLS

\begin{tabular}{|c|c|c|}
\hline a & $=$ & coefficient of thermal expansion $\left(\mathrm{m} / \mathrm{m} /{ }^{\circ} \mathrm{K}\right)$ \\
\hline $\mathrm{C}_{\mathrm{F}}$ & $=$ & Sound velocity in fluids $(\mathrm{m} / \mathrm{s})$ \\
\hline $\mathrm{C}_{\mathrm{L}}$ & $=$ & longitudinal velocity $(\mathrm{m} / \mathrm{s})$ \\
\hline $\mathrm{C}_{\mathrm{M}}$ & $=$ & mean integrated velocity $(\mathrm{m} / \mathrm{s})$ \\
\hline $\mathrm{C}_{\mathrm{O}}$ & $=$ & extensional velocity $(\mathrm{m} / \mathrm{s})$ \\
\hline $\mathrm{C}_{\mathrm{S}}$ & $=$ & shear velocity $(\mathrm{m} / \mathrm{s})$ \\
\hline $\mathrm{C}_{\mathrm{P}}$ & $=$ & specific heat at constant stress $\left(\mathrm{J} / \mathrm{kg} \cdot{ }^{\circ} \mathrm{K}\right)$ \\
\hline $\mathrm{E}$ & $=$ & Young's modulus $(\mathrm{Pa})$ \\
\hline $\mathrm{E}_{\mathrm{A}}$ & $=$ & adiabatic Young's modulus $(\mathrm{Pa})$ \\
\hline $\mathrm{E}_{\mathrm{I}}$ & $=$ & isothermal Young's modulus $(\mathrm{Pa})$ \\
\hline $\mathrm{f}$ & $=$ & frequency $(\mathrm{Hz})$ \\
\hline $\mathrm{G}$ & $=$ & shear modulus $(\mathrm{Pa})$ \\
\hline $\mathrm{h}$ & $=$ & Planck's constant $\left(662.60755 \times 10^{-36} \mathrm{~J} \cdot \mathrm{s}\right)$ \\
\hline $\mathrm{K}$ & $=$ & bulk modulus $(\mathrm{Pa})$ \\
\hline $\mathrm{k}$ & $=$ & Boltzmann's constant $\left(13.80658 \times 10^{-24} \mathrm{~J} /{ }^{\circ} \mathrm{K}\right)$ \\
\hline $\mathrm{h} / \mathrm{k}$ & $=$ & constant $\left(47.99216 \times 10^{-12}{ }^{\circ} \mathrm{K} \cdot \mathrm{s}\right)$ \\
\hline $\mathbf{M}$ & $=$ & atomic weight \\
\hline $\bar{M}$ & $=$ & mean atomic weight \\
\hline $\mathrm{N}$ & $=$ & Avogadro's number $\left(602.2167 \times 10^{21} \mathrm{gmol}^{-1}\right)$ \\
\hline $\mathrm{P}$ & $=$ & number of atoms per molecule \\
\hline $\mathrm{R}$ & $=$ & reflection coefficient \\
\hline $\mathrm{Z}$ & $=$ & acoustic impedance $\left(\mathrm{kg} / \mathrm{m}^{2} \cdot \mathrm{s}\right)$ \\
\hline$\varepsilon$ & $=$ & strain $(\mathrm{m} / \mathrm{m})$ \\
\hline$\theta$ & $=$ & Debye temperature $\left({ }^{\circ} \mathrm{K}\right)$ \\
\hline$\lambda$ & $=$ & wavelength (m/cycle) \\
\hline$\lambda^{\prime}$ & $=$ & Lame modulus $(\mathrm{Pa})$ \\
\hline$v$ & $=$ & Poisson's ratio \\
\hline$\rho$ & $=$ & density $\left(\mathrm{kg} / \mathrm{m}^{3}\right)$ \\
\hline$\sigma$ & $=$ & stress $(\mathbf{P a})$ \\
\hline
\end{tabular}




\section{DETERMINING ULTRASONIC VELOCITY ULTRASONIC TRANSDUCTION}

The measurement of ultrasonic velocities depends upon generating a dynamic pressure wave (pulse) into a material of known thickness and measuring the transit time of the emerging acoustic pressure wave. The generation and detection of an acoustic wave is usually accomplished by a piezoelectric transducer. The element is cut to a particular frequency, mode of operation (compressional, distortional, etc.), and is then assembled into a transducer case. The element has a measured amount of mechanical damping applied to the back surface. The transducers are purchased from commercial transducer manufacturers for the specific purpose of measuring the ultrasonic velocities with a high degree of resolution. Piezoelectric transducers are frequently used instead of magnetostrictive transducers because they are more adaptable to extreme ranges of frequency, have greater conversion efficiency and provide the greatest sensitivity. There are vast differences in frequency response, selectivity and sensitivity among the various materials used for their piezoelectric properties. Transducer manufacturers have discovered methods to significantly alter many transducer characteristics, such as, using mechanically coupled damping materials to prevent the excessive ringing following the initial excitation of the piezoelectric element. Many aspects of transducer fabrication rely on propriatary factors.

The shape of the pulse, generated by the electronic pulser, has a major influence on the pressure wave induced in the material. The input pulse is generally shaped to form a tail pulse in order to damp ringing following the initial pulse step. The ideal broadband pressure pulse should: (a) be a $(\sin \mathrm{x}) / \mathrm{x}$ function, since such a pulse can be shown to have an extremely wide bandwidth, and (b) have a well defined, characteristic peak. In practice, the $(\sin x) / x$ pulse is approximated by filtering the pulse spectrum at the spectrum extremes. The result of this attenuation is a time domain pulse of 5 to 7 half-cycles, with a well defined peak amplitude at the center. In the spectral domain, this waveform is shown bandpassed, resulting in the attenuation of both the lowest and highest frequencies, with a gradual peaking at the central frequency that, in turn, is proportional to the reciprocal of the period of the central peak in the time history. 


\section{REFLECTION OF THE ULTRASONIC WAVE}

Reflection of the ultrasonic wave occurs at the interface of two different acoustic impedances. ${ }^{2}$ The specific acoustic impedance is given in rayls $\left(\mathrm{kg} / \mathrm{m}^{3} \cdot \mathrm{s}\right)$. The acoustic impedance is defined for bulk materials where the sound propagation is normal to the transducer to specimen interface as $\rho \mathrm{C}_{\mathrm{L}}$, where $\rho$ is the mass density and $\mathrm{C}_{\mathrm{L}}$ is the longitudinal velocity of sound in that medium. However, the impedance is further multiplied by the cross-sectional area for materials that are small compared to the wavelength of the sound. Materials whose lateral dimensions fall between $0.2 \lambda$ and $5 \lambda$ are avoided since the resulting longitudinal velocity is not defined. The transducer-to-specimen interface is a planar surface that frequently involves two or more different acoustic impedances. This difference in impedances causes a phase shift of the reflected ultrasound under specific conditions. The general equation for the sound pressure Reflection Coefficient shows how the phase shift may occur.

$$
R=\frac{\left(Z_{2} / Z_{1}\right)-1}{\left(Z_{2} / Z_{1}\right)+1}
$$

This indicates that when the sound is reflected from an interface where the second medium has a lower acoustic impedance than the first medium, a $180^{\circ}$ phase shift occurs. The sound transmitted through an interface undergoes no phase shift, however. There may be varying amounts of phase shift from zero to several radians, depending upon the relative complex impedances involved in the reflection. This phase shift causes a discrepancy in the measured sound velocity, as described by McSkimin. 3,4 The phase shift is a result of the transit (round-trip) time for sound to penetrate through the couplant and through the piezoelectric element and transducer wear face.

The usual method of measuring the time between reflected ultrasonic echoes shows a disparity in the time interval between one pair of adjacent echoes compared with the time interval between any other pair of adjacent echoes in an echo train. The amount of error may be determined, and the velocity of sound thereby corrected, by a technique known as the Pulse Superposition Method. ${ }^{3-5}$

This technique was further refined and modified by Papadakis ${ }^{6,7}$ to include the correction for ultrasonic diffraction loss and phase change. This later technique replaced the former method of time interval, or echo repetition rate measurements, with a frequency 
measurement. The horizontal sweep on an oscilloscope is driven by an oscillator operating on a frequency whose period is equal to the time between echoes. The frequency is measured by a frequency counter, increasing the number of significant digits, which in turn increases the precision of the measurement. In addition, a tone burst generator permits a slight shift in the piezoelectric driving frequency that allows the operator to detect, and correct for, the amount of phase shift that has occurred within the specimen, thereby increasing the accuracy of measurement. This technique is amoung those used at LLNL for the determination of the ultrasonic velocities in lightly attenuating specimens that permit the ultrasound to form multiple echoes.

If the specimen is highly attenuating, multiple echoes cannot be detected and a single through-the-specimen pulse is measured for the time interval. The accuracy of the time interval measurement can be enhanced by an acoustic buffer or delay line. The accuracy can be only roughly estimated for velocities where extreme attenuation of sound causes difficulty in defining a separation of the pulse from the noise. In addition, the soundattenuating materials usually force the use of thin specimens and low frequency transducers, further degrading the accuracy of the measurements.

\section{DERIVATION OF SONIC VELOCITIES}

The derivation of the sonic velocities is a property of the elastic constants of materials. The analysis of the fundamental assumption of elasticity theory, which states that linear stress is proportional to linear strain,

$$
\sigma_{i j}=C_{i j k l} \varepsilon_{k l},
$$

is the origin of all further analysis. In general, it may be shown that for any prescribed direction of propagation three plane waves can exist. The direction of propagation is taken to be perpendicular to the planes of equal phase. The phase velocities $(V)$ for these waves may be obtained from the density $\rho$ and the elastic stiffness moduli by the use of the Christoffel relations. The number of physical properties may be substantially reduced, depending upon the crystallographic symmetries, according to Neuman's principle ${ }^{8,12}$ by a reduction of redundancies. An example of a simple cubic, single crystal is shown below. The required elements for isotropic materials are adapted from the simple cubic as illustrated. The directional arrows show the particle direction for the shear waves. 


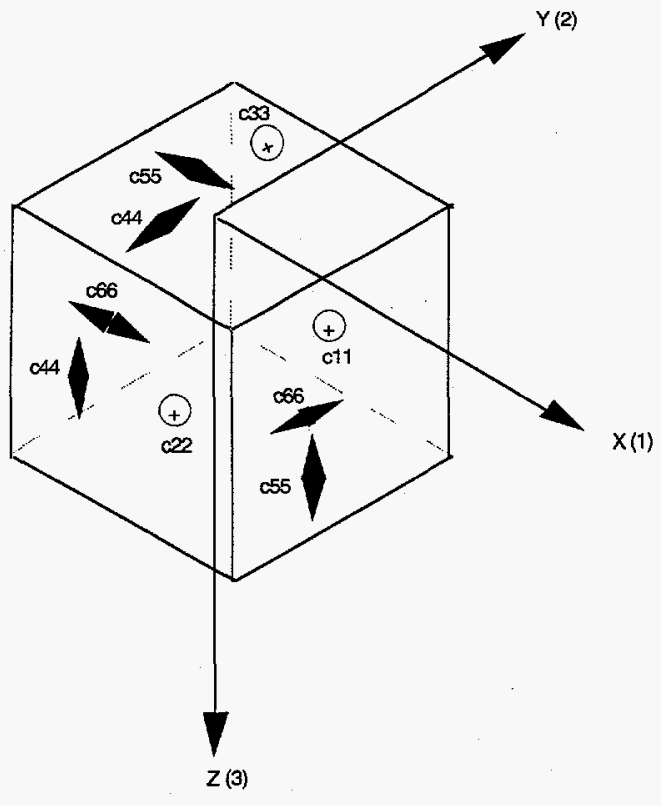

CUBIC UNIT CELL

$$
\begin{aligned}
& c_{11}=c_{22}=c_{33} \\
& c_{12}=c_{13}=c_{23} \\
& c_{44}=c_{55}=c_{66}
\end{aligned}
$$

Where:

$$
c_{12}=c_{11}-2 c_{44} \text { and } V^{2}=c_{i j} / \rho
$$

For isotropic materials, a simplification from three measurements to two measurements is performed as follows:

$$
c_{44}=c_{55}=c_{66}=0.5\left(c_{11}-c_{12}\right)
$$

The elastic constants are derived as follows ${ }^{8}: c_{11}=2 G+\lambda^{\prime}$ and $c_{12}=\lambda^{\prime}$. Converting stiffness constants to compliances, the values for Young's modulus, Poisson's ratio, and the shear modulus may be derived. $s_{11}=1 / E, s_{12}=-v / E$, and $2\left(s_{11}-s_{12}\right)=1 / G$. From these relations follows, $G=E /[2(1+v)]$. Where the compliances are, $s_{11}=\frac{c_{11}^{2}-c_{12}^{2}}{\left(c_{11}-c_{12}\right)\left\{c_{11}\left(c_{11}+c_{12}\right)-2 c_{12}^{2}\right\}}$ and $s_{12}=\frac{c_{12}^{2}-c_{12} c_{11}}{\left(c_{11}-c_{12}\right)\left\{c_{11}\left(c_{11}+c_{12}\right)-2 c_{12}^{2}\right\}}$.

Isotropic materials provide the greatest reduction in number of elastic moduli since for any direction through the material, the moduli are repeating. Anisotropic materials are frequently sent into the laboratory for ultrasonic velocity measurements, but it is seldom that such materials are sent in for moduli calculation. The primary reason for this is probably one of the following:

1. The crystallographic axis is not known.

2. Only one specimen of a single crystallographic orientation is available 
3. Cost or time is prohibitive.

4. The client submitting the specimen is only interested in knowing whether or not it is anisotropic.

For this reason, the rigorous analysis of elastic constants for anisotropic materials will not be described here, and the reader is referred to some excellent treatises on the subject. 1,8 The subject matter described here is concerned with the unique solutions of the basic matrix equations for isotropic materials. Indeed, the material requirements for these analyses are rigidly limited to both isotropic and homogeneous materials. The principal ultrasonic velocities measured at the laboratory are the infinite medium (bulk specimen) longitudinal and shear velocities, although the extensional velocity is measured, on occasion, on wires, fibers, and thin rods.

\section{SPECIMEN GEOMETRY}

The specimen geometry for a particular ultrasonic velocity determination may have a decided effect on the wave propagation mode and on the measured wave speed. Generally, velocity determinations are more accurate on relatively thick specimens, for three reasons:

1. As the thickness of the specimen increases, so does the number of significant digits to which one can accurately measure the thickness.

2. The effect on specimen geometry of a short time interval change in ambient temperature decreases with thickness due to the greater specimen volume (thermal inertia).

3. The pulsed ultrasound pressure wave has finite dimensions, generallized as a relatively small diameter cylinder or cone, roughly approximating the diameter of the transducer within the near field and spreading conically in the far field to: $\sin \frac{\theta}{2}=0.514 \frac{\lambda}{D}$, where D is the transducer diameter: Calculated for the $-6 \mathrm{~dB}$ down amplitude for flat transducers.

4. The first maxima back from the extreme far field $\left(\mathrm{Y}^{+} \mathrm{O}\right)$ is given as $S_{C}=\frac{Z \lambda}{a^{2}}=\frac{Z C_{L}}{a^{2} f}=1$, where $\mathrm{Z}$ is the distance along the ultrasonic beam, $\mathrm{C}_{\mathrm{L}}$ is the longitudinal velocity of the medium, $a$ is the transducer radius, $\lambda$ is the wavelength, and $f$ is the frequency in Hertz. The start of the far field in the ultrasonic beam profile is approximated as: $\frac{D^{2} f}{4 C_{L}}$, where $\mathrm{D}$ is the transducer diameter. 
The induced pressure wave should involve no more than one-sixth of the smallest dimensions of a specimen, depending upon the desired wave propagation mode. While it can be shown, one may intuitively see that as a sound pressure wave interacts with the specimen boundaries, tension, compression and shear forces contend for position, with the result that during this convolution of waves a severe distortion and interference of the waves occurs, rendering accurate measurement of time of arrival of these waves difficult. Most materials tend to perform as mechanical filters to ultrasound. Most generally, the materials behave like low pass filters, which attenuate the amplitude of the pressure waves in proportion to the increasing frequency and the increasing thickness in the direction of the wave propagation. However, attenuation is also a function of many other phenomena inherent in the materials, see Appendix B. Losses in solids are mainly the result of heat conduction, viscous friction, dislocation motion, and scattering. Losses in high-polymer materials (plastic, rubber, etc.) is mainly of viscous nature. It is therefore neither practical nor possible to set down a fixed specimen geometry beyond stating that the specimen may have flat, parallel faces with a parallelism of better than $25 \times 10^{-5}$ (in./in., $\mathrm{m} / \mathrm{m}$, etc.) for many polycrystalline materials, and to about $10^{-6}$ for single-crystal specimens.

In addition, for most materials the diameter, and for rectangular specimens the edge dimension, should be three times the thickness (an aspect ratio of $\geq 3: 1$ ). The thickness is usually 3 to $25 \mathrm{~mm}$, depending upon the attenuation properties of the material. Sometimes these dimensions are radically altered, as for example, when the material is found only in the shape of rods, or wires, or even a textile yarn. A specialized geometry is required when the acoustic velocities are desired as a function of stress, strain, or temperature. Proper specimen preparation is essential for the determination of specific ultrasonic velocities.

\section{VELOCITIES: LONGITUDINAL AND SHEAR WAVES}

\section{Longitudinal Velocities}

The first velocity usually determined is the longitudinal, often referred to as compressional, nondistortional, $\mathrm{L}$ wave, $\mathrm{P}$ wave, waves of dilatation, and irrotational waves. The longitudinal velocity is specimen-geometry to wavelength dependent. Thin bars, rods, or wires will propagate a longitudinal wave at a lower velocity than the same material of infinite dimension. The first observation of this difference in velocities shows a strong wavelength dependence. When the wavelength $(\lambda=C / f)$ is less than one-fifth of the solid specimen's lateral dimensions along the path of wave propagation, the specimen is often referred to as a bulk specimen, and the velocity is often referred to as a bulk specimen 
longitudinal velocity. This is the velocity most usually measured at the laboratory, and it has a definite relationship to the elastic properties of a solid isotropic homogeneous material:

$$
C_{L}=\sqrt{\frac{E}{\rho}\left[\frac{1-v}{(1+v)(1-v)}\right]}
$$

$E=$ Young's Modulus, $\rho=$ density, $v=$ Poisson's ratio.

\section{Shear Velocities}

The shear wave appears to be the most easily propagated waveform in nature and usually has the highest amplitude. Shear waves predominate in seismic activities and in all forms of fracturing. Typically, shear waves exhibit amoung the lowest frequency of vibration of sound within a specimen and amoung the greatest displacement of material. Shear waves are often called transverse waves, distortional waves, rotational waves, torsional waves, and $S$ waves. They are usually the most difficult kind of wave to generate with transducers, and since the shear velocity is approximately one-half the velocity of longitudinal waves, the shear wavelength is on the order of one-half the wavelength of longitudinal waves for the same frequency. For this reason it is frequently necessary to use a shear wave frequency that is one-half the maximum longitudinal wave frequency. Some materials may support a longitudinal wave to the exclusion of shear waves, but any material that supports a shear wave will support a longitudinal wave. All other wave velocities, e.g., Stonely, Lamb, Rayleigh, Love waves, and thin rod or bar longitudinal waves, can exist only because the material can sustain both a shear and longitudinal wave. Materials that cannot sustain a shear wave, or shearing force, must be materials that are perfectly fluid, i.e., have zero viscosity. The shear velocity, $\mathrm{C}_{S}$, is related to the shear modulus in isotropic homogeneous materials by

$$
C_{S}=\sqrt{G / \rho}
$$

where $G$ is the shear modulus and $\rho$ is the density.

The particle direction of shear waves generated by shear wave transducers is always normal to the propagation direction. Shear waves are divided by usage into two types, $S_{v}$ and $S_{H}$, called shear vertical and shear horizontal, depending upon the particle direction with respect to the propagation direction. $S_{H}$ waves are usually introduced into a material through a wedge such that the particle direction is in the plane of the material. The $S_{H}$ wave will not 
mode convert to a longitudinal signal, but remain as a pure shear wave. The $S_{\mathrm{v}}$ wave may also be introduced into a material through a wedge, but the particle direction is perpendicular to the propagation of the $S_{H}$ wave. As $S_{v}$ the wave just touches the material, part of the wave enters the material at the same moment that it is still partly in the wedge. This $\mathrm{S}_{\mathrm{v}}$ wave will mode convert according to Snell's law, ${ }^{9}$ which states

$$
\frac{\sin \theta_{S}}{C_{S}}=\frac{\sin \theta_{L}}{C_{L}}=\frac{\sin \theta_{L}^{\prime}}{C_{L}^{\prime}}=\frac{\sin \theta_{S}^{\prime}}{C_{S}^{\prime}}
$$

where:

$$
\begin{aligned}
& \theta_{S}=\text { shear angle in the first medium, } \\
& \theta_{L}=\text { longitudinal angle in the first medium, } \\
& C_{S}=\text { shear velocity in the first medium, } \\
& C_{L}=\text { longitudinal velocity in the first medium, } \\
& \text { and the primed values refer to the second medium. }
\end{aligned}
$$

This is the same condition that exists when a longitudinal wave is introduced into a material at an angle. Angles are referenced to the axis-normal (perpendicular) to the plane of the specimen. Note that there is no refracted critical angle when the sound velocity in the first medium is greater than the sound velocity in the second medium. When the longitudinal sound velocity in the first medium is less than the sound velocity in the second medium, the incident longitudinal wave angle will be: $\theta_{1}=\sin ^{-1}\left(\frac{C_{1}}{C_{2}} \sin \theta_{2}\right)$, and when the refracted longitudinal wave is at the first critical angle, the refracted shear wave $\left(S_{\mathrm{v}}\right)$ angle will be: $\theta_{2}=\sin ^{-1} \sqrt{(1-2 v) / 2(1-v)}$.

The $S_{\mathrm{v}}$ wave also results when a shear-wave transducer at normal incidence is coupled with a viscous, sticky, resinous coupling agent to a material capable of sustaining shearing forces.

\section{COUPLING THE TRANSDUCER TO THE SPECIMEN}

The ultrasonic transducer must be coupled to the material in such a manner as to exclude the presence of air voids. With shear waves, coupling is even more critical than with longitudinal waves. Coupling may be accomplished through pressure if the transducer face and material have excellent mating surfaces, similar to those of gage blocks. Transducers are sometimes coupled to specimens with adhesives or low-melting-temperature salts, but easier methods are usually available. Specimens may react to certain coupling agents or 
otherwise adversely affected by absorption; in these cases, coupling can sometimes be accomplished by a combination of pressure and a sheet of Saran Wrap@ sandwiched between the specimen and the transducer. The usual shear wave couplant is a modified resin without a catalyst. This resin has a high viscosity at or below room temperature, but thins rapidly as the temperature increases. It works well for longitudinal waves as well as shear waves.

\section{RELATIONSHIP BETWEEN ULTRASONIC VELOCITY AND ELASTIC MODULI}

\section{YOUNG'S MODULUS}

By virtue of symmetry, the relationship of the ultrasonic velocities to the tangent modulus is found for isotropic homogeneous materials from a simplification of the 36 possible elastic constants derived for anisotropic materials. The formulae for isotropic homogeneous materials is shown below. The longitudinal velocity is given by

$$
C_{o}=\sqrt{\frac{Y_{o}}{\rho}}=\sqrt{\frac{(1-v)}{(1+v)(1-2 v)}} \text { when the wavelength of the propagating ultrasound }
$$

is greater than five times the diameter of the rod or bar. The effective stiffness modulus $\mathrm{Y}_{\mathrm{L}}$ of such a constrained ${ }^{1}$ section will be higher than the Young's modulus $Y_{O}$ for a free bar.

$$
C_{L}=\sqrt{Y_{L} / \rho}=\sqrt{\frac{Y_{O}}{\rho(1-b)}} \text { where the formula for } Y_{O} \text { takes into account the }
$$

restraining forces for $\mathrm{C}_{\mathrm{L}} \cdot \frac{Y_{o}}{Y_{L}}=1-b$, and $b=2 v^{2} /(1-v)$, with the limit of $\mathrm{b}=1$ when $v=0.5$. The stiffness modulus is a complex number with the imaginary part corresponding to the loss factor. When the loss factor is small, it is usually ignored and the stiffness modulus is reported as a scaler number.

$$
C_{L}=\sqrt{(K+(4 / 3) G) / \rho}
$$

where $\mathrm{K}=$ bulk modulus, $\mathrm{G}=$ shear modulus, and

$$
C_{L}=\sqrt{(E / \rho)[(1-v) /((1+v)(1-2 v))]}
$$

\footnotetext{
${ }^{1}$ The propagating ultrasound through the length of a bar will cause the lateral dimensions to change, dynamically, with respect to the position of the pressure wave within the bar. A material with infinite lateral dimension does not constrain the lateral edges of the longitudinal stress wave.
} 
where $v=$ Poisson's Ratio. When $v$ is $1 / 2, C_{L}$ is indeterminate.

However, Young's modulus is equivalent to

$$
E=3 K(1-2 v)
$$

Now, by substituting Eq. (6) into Eq. (5), a condition may be established that will hold for an ideal, simple fluid, i.e., a fluid with no viscosity. First let

$$
3(1-2 v)(1-v) /[(1+v)(1-2 v)]=1
$$

The solution of Eq. (7) yields

$$
\begin{aligned}
& 3(1-2 v)=1+v \\
& 2=4 v \\
& v=1 / 2
\end{aligned}
$$

Therefore, since $v=1 / 2$, the material meets the fluid requirement, and the substitution for velocity can be completed for the perfect fluid condition

$$
C_{F}=\sqrt{K / \rho}
$$

which describes the velocity of sound through many liquids and gases. The shear velocity is found in materials that sustain shearing forces, such as most solids and many viscous fluids. The shear velocity of sound is given by

$$
C_{s}=\sqrt{\frac{G}{\rho}}
$$

Now, one may mistakenly combine Eqs. (8) and (9) and substitute the result back into Eq. (4), which would then yield

$$
C_{L}=\sqrt{\rho\left[C_{f}-(4 / 3) C_{s}^{2}\right] / \rho}=\sqrt{C_{f}^{2}-(4 / 3) C_{s}^{2}}
$$

However, the earlier requirement placed upon the validity of Eq. (8) will be violated if the validity requirement for Eq. (9) remains extant, that is, a material may not be a perfect fluid and sustain shearing forces. It is left to the reader to conjecture whether Eq. (10) could prevail immediately after the detonation of a high explosive into a solid material, causing that material to become totally fluid for an instant. It is not likely, however, that the material could sustain shearing forces during that instant in time. There is another 
longitudinal sonic velocity that is encountered in materials capable of sustaining a shearing force. This longitudinal velocity is called the extensional, bar, or thin rod velocity. Rods, bars, or wires that are subjected to a compressional wave with a wavelength greater than five times the cross sectional dimension of the rod permits the wave to propagate at a reduced velocity compared with the bulk specimen longitudinal wave. This velocity, $\mathrm{C}_{\mathrm{O}}$, is related to Young's modulus by Eq. (11).

$$
C_{o}=\sqrt{E / \rho}
$$

and is either calculated from $\mathrm{C}_{\mathrm{L}}$ and $\mathrm{C}_{\mathrm{S}}$ or measured from specially designed specimens and transducers. By solving Eq. (9) for the shear modulus,

$$
G=C_{S}^{2} \rho
$$

and by substituting this equation into Eq. (4), the bulk modulus is calculated:

$$
C_{L}^{2}=\left[K+(4 / 3) C_{S}^{2} \rho\right] / \rho
$$

and

$$
K=\rho\left[C_{L}^{2}-(4 / 3) C_{s}^{2}\right]
$$

The relationship of Young's modulus to the bulk and shear modulus is

$$
E=9 K G /(G+3 K)
$$

If Eq. (13) is substituted for K, and Eq. (12) is substituted for G in Eq. (14), Young's modulus becomes

$$
E=\rho C_{S}^{2}\left(3 C_{L}^{2}-4 C_{S}^{2}\right) /\left(C_{L}^{2}-C_{S}^{2}\right)
$$

and by substituting Eq. (15) into Eq. (11), the extensional velocity becomes

$$
C_{o}=\sqrt{C_{S}^{2}\left(3 C_{L}^{2}-4 C_{S}^{2}\right) /\left(C_{L}^{2}-C_{S}^{2}\right)}
$$

The dependence of the extensional velocity on a shear velocity is apparent and is consistant with the concept that materials that cannot support shearing forces, such as fluids with zero viscosity, cannot propagate an extensional velocity. The extensional velocity may be visualized as a compressional pressure wave that exhibits a maniscus effect on the specimen walls as the wave propagates along the rod axis. 


\section{POISSON'S RATIO}

It is axiomatic that the elastic constants are all interrelated. Therefore, once the isotropic moduli are established, Poisson's Ratio may be calculated. Poisson's Ratio is also a function of the ratio of the longitudinal and shear velocities,

$$
v=\left[1-2\left(C_{S} / C_{L}\right)^{2}\right] /\left[2-2\left(C_{S} / C_{L}\right)^{2}\right]
$$

and a set of curves is shown in the Nondestructive Testing Handbook ${ }^{21}$, depicting the ratio of any two of four ultrasonic velocities as a function of Poisson's Ratio.

\section{LAME' CONSTANTS}

There are two Lame elastic constants calculated from the ultrasonic-velocities. One of the Lame constants has been given as the shear modulus. The other is sometimes listed as the Lame' modulus, $\lambda^{\prime}$. The Lame' modulus ${ }^{10}$ is a governing factor for both the longitudinal and shear velocities:

$$
C_{L}^{2}=\left(\lambda^{\prime}+2 G\right) / \rho
$$

and by rearranging the formula, the Lame' modulus is

$$
\lambda^{\prime}=\rho\left(C_{L}^{2}-2 C_{S}^{2}\right)
$$

The Lame modulus is perhaps of interest to those who work with plastic materials. As Poisson's Ratio increases, the Lame modulus will numerically approach the bulk modulus. The shear modulus will disappear as the viscosity of the fluid approaches zero. This may be observed from the recognition of the equation for bulk modulus: $K=\lambda^{\prime}+\frac{2 \mu}{3}$, where shear approaches zero for fluids and, simultaneously, Poisson's ratio approaches 0.5 . The compressional velocity for fluids is proportional to the bulk modulus as shown in equation 8.

\section{DEBYE TEMPERATURE}

The thermal loss mechanisms (temperature dependence) of materials is most suitably described in terms of the Debye temperature, $\theta$. The Debye temperature may be calculated with the help of the infinite medium, shear, and longitudinal velocities. The mean integrated velocity 5 is calculated for the isotropic material and then fed into an equation 
containing Planck's constant, Boltzmann's constant, Avogadro's number, the mean atomic weight, and the density of the material. The mean integrated velocity, $\mathrm{C}_{\mathrm{M}}$, is given by

$$
C_{M}=\left[3\left(C_{S} C_{L}\right)^{3} /\left(2 C_{L}^{3}+C_{S}^{3}\right)\right]^{1 / 3}
$$

and the Debye temperature, $\theta$, is

$$
\theta=(h / k)(3 P \rho N / 4 \pi \bar{M})^{1 / 3} C_{M}, \text { (Degrees Kelvin) }
$$

Where:

$\mathrm{h} / \mathrm{k}=$ Planck's constant $/$ Boltzmann's constant $=47.99216 \times 10^{-12}{ }^{\circ} \mathrm{K} \cdot \mathrm{s}$, $\mathrm{P}=$ Number of atoms per molecule,

$\mathrm{N}=$ Avogadro's number $\left(602.2167 \times 10^{21} \mathrm{kmol}^{-1}\right)$

$\bar{M}=$ Mean atomic weight.

\section{ADIABATIC VERSUS ISOTHERMAL YOUNG'S MODULUS}

Ultrasonic velocity measurements in material testing do not allow sufficient time for thermal diffusion. Therefore, Young's modulus, as calculated from the ultrasonic velocities, is the adiabatic modulus, ${ }^{1}$ which is slightly higher than the isothermal modulus. The relationship between the adiabatic and isothermal Young's modulus is given by:

$$
\begin{aligned}
& E_{A} / E_{i}=1+a^{2} T\left(E_{A} / \rho C_{P}\right) \\
& E_{i}=E_{A} /\left[1+\left(a^{2} T\left(E_{A} / \rho C_{P}\right)\right)\right] \\
& E_{A}=E_{i} \rho C_{P} /\left(\rho C_{P}-E_{i} a^{2} T\right)
\end{aligned}
$$

Where:

$a=$ coefficient of thermal expansion, $\mathrm{m} / \mathrm{m} /{ }^{\circ} \mathrm{K}$

$\mathrm{C}_{\mathrm{p}}=$ specific heat at constant stress, $\mathrm{J} / \mathrm{kg} \bullet^{\circ} \mathrm{K}$

$\mathrm{T}=$ absolute temperature

$\rho=$ density

$\mathrm{E}_{\mathrm{A}}=$ adiabatic Young's modulus

$\mathrm{E}_{\mathrm{i}}=$ isothermal Young's modulus 


\section{WHAT ELSE? A LOOK AT SOME OTHER TECHNIOUES}

A particularly useful technique for measuring ultrasonic velocities in a variety of materials at elevated temperatures was established at the laboratory by Dunegan ${ }^{11}$ from a compilation of experiments performed by McSkimin, ${ }^{12}$ and Reynolds. ${ }^{13}$ This technique required special machining of the specimens into two concentric continuous solid cylinders of different diameters and lengths. The larger diameter cylinder was threaded with 32 threads per inch to prevent interfering reflections from the circumference. This specimen allowed simultaneous measurement of the longitudinal and shear velocities from very low temperatures to about $870^{\circ} \mathrm{C}$. Special furnaces and cooling collars would have to be assembled before the test could be repeated. The technique worked out by Reynolds is shown on the following page: 


\section{REYNOLDS METHOD FOR DETERMINING SHEAR VELOCITIES}

The rod should be machined as a smooth, solid, right-circular cylinder with perpendicular and parallel faces. Solve for the shear velocity through transcendental equations.

Nominal dimensions for most metals are $16 \mathrm{~mm}$ diameter by $64 \mathrm{~mm}$ long.

$$
\begin{aligned}
& \text { Th } \sin \theta_{1}=1 \therefore \sin \theta_{2}=\frac{C_{S}}{C_{L}} \text { from } \frac{\sin \theta_{1}}{C_{L}}=\frac{\sin \theta_{2}}{C_{S}}
\end{aligned}
$$

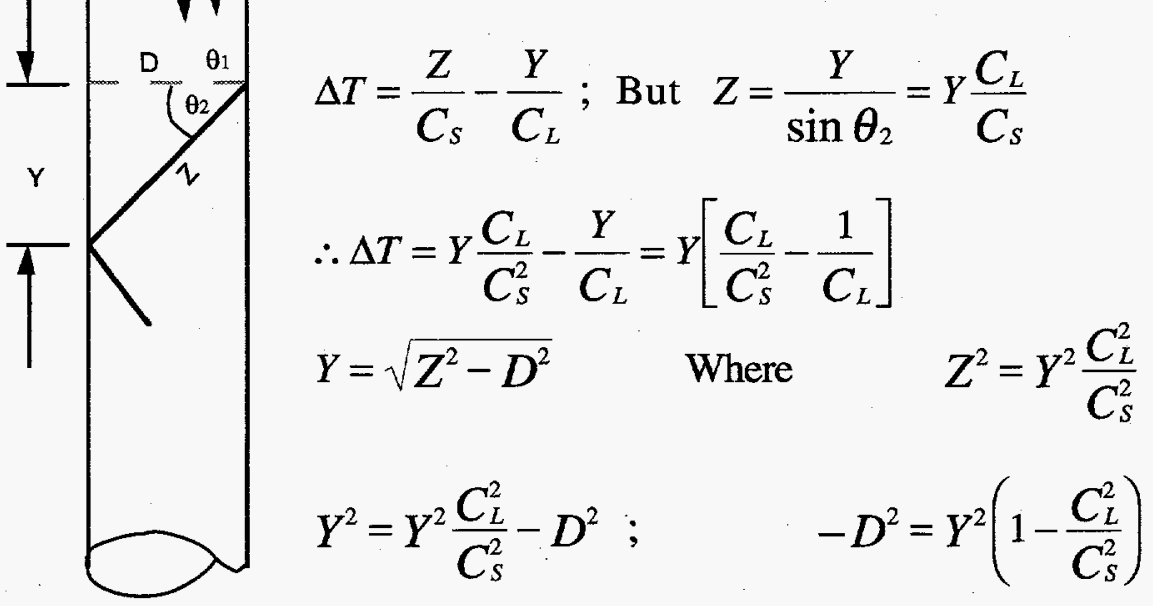

$$
Y^{2}=\frac{-D^{2}}{1-\frac{C_{L}^{2}}{C_{S}^{2}}}=\frac{D^{2}}{\frac{C_{L}^{2}}{C_{S}^{2}}-1}=\frac{D^{2} C_{S}^{2}}{C_{L}^{2}-C_{S}^{2}} \therefore Y=\frac{D C_{S}}{\sqrt{C_{L}^{2}-C_{S}^{2}}}
$$

Substitute (b) into (a):

$$
\begin{aligned}
& \Delta T=\frac{D C_{S}}{\sqrt{C_{L}^{2} C_{S}^{2}}}\left[\frac{C_{L}}{C_{S}^{2}}-\frac{1}{C_{L}}\right]=\frac{D C_{S}}{\sqrt{C_{L}^{2}-C_{S}^{2}}}\left[\frac{C_{L}^{2}-C_{S}^{2}}{C_{S}^{2} C_{L}^{2}}\right] \\
& \Delta T=\frac{D \sqrt{C_{L}^{2}-C_{S}^{2}}}{C_{L} C_{S}} ; \quad C_{L}^{2} C_{S}^{2} \Delta T^{2}=D^{2} C_{L}^{2}-D^{2} C_{S}^{2} \\
& C_{S}^{2}\left(C_{L}^{2} \Delta T^{2}+D^{2}\right)=D^{2} C_{L}^{2} ; \quad C_{S}^{2}=\frac{D^{2} C_{L}^{2}}{D^{2}+C_{L}^{2} \Delta T^{2}}
\end{aligned}
$$




$$
C_{S}^{2}=\frac{C_{L}^{2}}{1+\frac{C_{L}^{2} \Delta T^{2}}{D^{2}}} \therefore C_{S}=\frac{C_{L}}{\sqrt{1+\frac{C_{L}^{2} \Delta T^{2}}{D^{2}}}}=\frac{C_{L}}{\sqrt{1+\left(\frac{C_{L} \Delta T}{D}\right)^{2}}}
$$

\section{$C L \quad \Delta T$}

Ultrasonic pulse echo waveform showing the initial pulse with longitudinal echoes and $\Delta$ Ts.

In another technique, the ultrasonic velocities were measured through the various axes of $\mathrm{BeO}, \mathrm{ZnO}$, and $\mathrm{CdSe}$, hexagonal single crystals, for the purpose of calculating the elastic constants. ${ }^{14}$ This technique was adopted from work performed by McSkimin ${ }^{12}$ and involved the measurement of velocities through very small single crystals mounted to the end of a special quartz buffer rod. The longitudinal velocity was also measured with the aid of a water collimator-transducer system. These measurements used a tone burst generator with an adjustable pulse duration. The frequency tuning and long pulse duration resulted in ultrasonic echo interferometry. Constructive and distructive interference alternated with an increasing frequency as a result of specimen resonance.

Acoustic analysis of solid materials catagorized as thin rods, thin plates, or thin bars is often performed somewhat differently from the earlier discription on bulk materials. Sound generated into thin material may have a wavelength greater than five times the smallest material dimension. A typical test material might be wires. An analysis of the wave properties in uniform diameter thin materials such as wire is shown below:

\section{ULTRASONIC REFLECTIONS OF PRESSURE WAVES BASED UPON ACOUSTIC IMPEDANCE BETWEEN A BUFFER AND A SPECIMEN}

Materials that are available as thin rods or wires are uniquely suited to magnetostrictive delay line applications. Some anisotropic or non-homogeneous materials available as thin rods, such as solid rocket propellant or samples of wood or bone, may be evaluated using the magnetostrictive delay line technique. The magnetostrictive induced ultrasound will permit comparisons between similar materials. In addition, this technique may be used in the most hostile of environments provided that the specimen remains intact during the measurement of the ultrasonic propagation velocities. 
The magnetostrictive delay line technique is used for the measurement of both extensional (compressional) and torsional (shear) wave velocities. The propagation of these waves is non-dispersive if the wavelength is greater than five times the diameter of the thin rod. The two velocities are then used, together with the mass density, to derive the various moduli. In order to receive the two interface reflections (delay line to specimen, and specimen to air) with nearly equal amplitudes for both wave velocities, a solution to a binomial equation is required. Let the delay line to specimen interface reflection be " $\mathrm{A}$ " amplitude, and let the specimen to air reflection be "B" amplitude and " $\mathrm{C}$ " the second echo from the specimen to air interface. The assumptions for the boundary conditions may be found in "Pressure Reflectivity and Transmissivity.

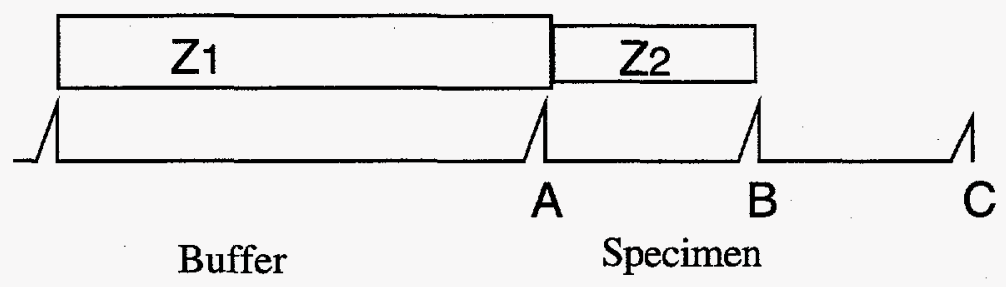

As a guide, the following is generally true for most metal specimens that are bonded to the magnetostrictive delay line. The following summary will be developed later in this paper. For extensional waves, the echo amplitudes from the specimen/delay line interface and from the end of the specimen, $\mathrm{A}=\mathrm{B}$ when the specimen diameter is approximately half the line diameter. Then, $\mathrm{A} / \mathrm{B}=4$ for torsional waves. Try for $(\mathrm{A} / \mathrm{B})$ ext. $=(\mathrm{B} / \mathrm{A})_{\text {tors. }}$, and the specimen diameter will be about 0.5 times the delay line diameter for extensional waves and about 0.7 times the delay line diameter for torsional waves. The optimum diameter ratio for extensional waves is $2: 1$, and the optimum diameter ratio for torsional waves is $2^{1 / 2}: 1$, or 1.4:1. The average for the two calculations will be about 0.6 times the delay line diameter. Non-metal specimens may have diameters approximating the diameter of the delay line. The optimum impedance ratio is the governing factor for establishing the optimum echo amplitudes. The length of a specimen should approximate an integer multiple of a half wavelength.

This analysis is based upon an ultrasonic transducer that is attached to one end of a buffer rod and the opposite end of the buffer rod is attached to a specimen. The acoustic impedance of the buffer is $Z_{1}$; the specimen is $Z_{2}$. The Reflection Coefficient $(R)$ and the Transmission Coefficient $(\mathrm{T})$ corresponds to the pressure wave distribution on the outgoing wave. The returning pressure waves, upon encountering the acoustic interfaces, have a Reflection Coefficient of $\left(\mathrm{R}^{\prime}\right)$ and a Transmission Coefficient of $\left(\mathrm{T}^{\prime}\right)$. 
Let: The acoustic impedance ratio for pressure waves: $\quad \boldsymbol{I}=\boldsymbol{Z}_{2} / \boldsymbol{Z}_{1}$

Then: $\boldsymbol{R}=(\boldsymbol{r}-1) /(\boldsymbol{r}+1) ; \quad \boldsymbol{T}=2 \boldsymbol{r} /(\boldsymbol{r}+1) ; \boldsymbol{T}=1+\boldsymbol{R}$

$$
\boldsymbol{R}^{\prime}=(1-\boldsymbol{r}) /(1+\boldsymbol{r}) ; \boldsymbol{T}^{\prime}=2 /(1+\boldsymbol{r}) \text {; }
$$

$\boldsymbol{T}^{\prime}=1+\boldsymbol{R}^{\prime}=1-\boldsymbol{R}$

The first interface echo, $A_{1}$, returning from the buffer-specimen interface, and the subsequent echoes $A_{2} \cdots A_{N}$ (where $N$ is the echo number) returning from the free end of the specimen are related in amplitude by the corresponding acoustic impedances, $\left(Z_{1}, Z_{2}\right)$ so long as the echoes are non-interfering with each other. The echo relative amplitudes, assuming that the attenuation is negligibly small, are equal to:

$$
\begin{aligned}
& \boldsymbol{A}_{1}=\boldsymbol{R} \\
& \boldsymbol{A}_{2}=\left(1-\boldsymbol{R}^{2}\right)=(1-\boldsymbol{R})(1+\boldsymbol{R})=\boldsymbol{T} \boldsymbol{T}^{\prime} \\
& \boldsymbol{A}_{3}=\boldsymbol{R}^{\prime}\left(1-\boldsymbol{R}^{2}\right) \\
& \boldsymbol{A}_{\boldsymbol{N}}=\boldsymbol{R}^{\prime N-2} \boldsymbol{T} \boldsymbol{T}^{\prime} \quad \text { Where } \mathrm{N} \text { is an integer } \geq 3
\end{aligned}
$$

The end echo of a series of repeating acoustic interfaces will be proportional to $\pm R^{\prime N} T T^{\prime}$, depending upon whether the termination is "open" or "short" circuited, respectively. If the attenuation is considered, the correction for the attenuation coefficient in nepers per unit length, $\alpha$, is $\exp (-2 \alpha L) A_{2} ; \exp (-4 \alpha L) A_{3}$. Also, see Appendix C.

Where: $\alpha=\ln \left(\left|\boldsymbol{R} \boldsymbol{A}_{2} / \boldsymbol{A}_{3}\right|\right) / 2 \boldsymbol{L}$.

The ability to match the reflected amplitudes of the echoes returning from the bufferspecimen interface with the echo returning from the free end of the specimen would be desirable. Small changes in the relative diameters of the buffer or the specimen will result in large changes in the acoustic impedance, resulting in large and unconstrained variations in the echo amplitudes. Such amplitude variations can cause one echo to saturate and at the same time put the other echo below the noise threshold. Thin rod, or wire, technology in the application of ultrasound easily avails itself to adjustment of the acoustic impedance. As long as the compressional wavelength is greater than five times the diameter of the buffer and the specimen, the waves will propagate without dispersion. The relationship of the diameter (D) to wavelength $(\lambda)$ may be shown as follows:

$$
D / \lambda_{0} \leq 0.2 \quad D / \lambda_{t} \leq 1.2
$$


Where $\lambda$ is the acoustic velocity divided by the acoustic frequency and the subscript denotes the extensional $(\mathrm{o})$, or the torsional $(\mathrm{t})$ wave mode.

The cross sectional area or the polar moment of inertia, depending upon whether the wave is propagating as longitudinal (extensional) or shear (torsional), respectively, influences the acoustic impedance. For buffers and specimens that can be described as solid right circular cylinders, the acoustic impedance is:

$$
\begin{array}{ll}
z_{0}=\pi \rho C_{0} D^{2} / 4 & \text { (Extensional waves) } \\
z_{t}=\pi \rho C_{t} D^{4} / 32 & \text { (Torsional waves) }
\end{array}
$$

Where $\rho$ is the density, $\mathrm{D}$ is the diameter, and $\mathrm{C}$ is the respective sound velocity. The impedance for torsional waves take into account the polar moment of inertia for a solid right circular cylinder, rather than the cross sectional area used to determine the impedance for extensional waves. Notice the strong effect the diameter has on the acoustic impedance.

The determination of the optimum reflection coefficient may proceed from the reflected intensity relationship and the solution of the quadratic equation as follows:

Let:

$$
\boldsymbol{r}=\boldsymbol{z}_{2} / \boldsymbol{z}_{1}
$$

Then: $\quad R=[(r-1) /(r+1)]^{2}$

$$
\begin{aligned}
& \boldsymbol{R}+\boldsymbol{T}=1 \\
& \boldsymbol{A}=\boldsymbol{I}_{\boldsymbol{O}} \boldsymbol{R} \\
& \boldsymbol{B}=\boldsymbol{I}_{0} \boldsymbol{T}^{2}
\end{aligned}
$$$$
\text { (buffer/specimen interface echo amplitude) }
$$
(specimen free end echo amplitude)

Let:

$$
A=B
$$

(equal amplitude reflections)

$$
\begin{aligned}
& I_{0} \boldsymbol{R}=\boldsymbol{I}_{0} \boldsymbol{T}^{2} \\
& \boldsymbol{T}^{2}=(1-\boldsymbol{R})^{2} \\
& \boldsymbol{I}_{0} \boldsymbol{R}=\boldsymbol{I}_{0}(1-\boldsymbol{R})^{2}=\boldsymbol{I}_{0}\left(\boldsymbol{R}^{2}-2 \boldsymbol{R}+1\right) \\
& \boldsymbol{R}=\boldsymbol{R}^{2}-2 \boldsymbol{R}+1
\end{aligned}
$$

Subtract R: $\quad 0=\boldsymbol{R}^{2}-3 \boldsymbol{R}+1$

\section{QUADRATIC SOLUTION:}

$$
\begin{array}{ll}
\boldsymbol{R}=\left(3 \pm 5^{1 / 2}\right) / 2=2.618 ; 0.382 & \text { (wave intensity) } \\
\boldsymbol{R}^{1 / 2}=(\boldsymbol{r}-1) /(\boldsymbol{r}+1)= \pm 1.619 ; \pm 0.618 & \text { (wave pressure) }
\end{array}
$$

Solve For $\mathrm{r}$ in Terms of $\mathrm{R}$ :

$$
\begin{array}{ll}
\boldsymbol{r} & =\left(1+\boldsymbol{R}^{1 / 2}\right) /\left(1-\boldsymbol{R}^{1 / 2}\right) \\
\boldsymbol{r} & =\boldsymbol{z}_{2} / \boldsymbol{z}_{1}= \pm 4.236 ;
\end{array}
$$


Only the positive roots are used since the negative roots are imaginary.

Note: Buffer-Specimen relationship where the buffer diameter is equal to or greater than 30 $\lambda: 2 \mathrm{~L} / \mathrm{C}_{\text {BUFFER }}=\mathrm{N}(2 \mathrm{thk}) / \mathrm{C}_{\text {SPECIMEN }}$, for bulk specimens also holds for thin rods with one exception. Where $\mathrm{N}$ is the number of the echo; thk is the thickness of the specimen, and $\mathrm{L}$ is the length of the buffer. In the application of the buffer to thin rod wave technology, the large diameter buffer is not required. The buffer, or delay line, may be adjusted in diameter to match the impedance of the magnetostrictive line on the one end and the specimen on the other end. A tapered delay line is sometimes referred to as a transformer, or impedance transformer.

\section{PRESSURE REFLECTIVITY AND TRANSMISSIVITY ${ }^{2}$}

The assumption for the following analysis is that the waves are generated in the first medium and travel into the second medium. Some of the pressure signal is reflected at the interface of the two mediums, but once entering the second medium, the pressure signal propagates without reflection. Assume the boundary at $\boldsymbol{X}=0$, so that the exponentials $e^{ \pm j k x}=1$. The total pressure is the same on both sides of the boundary; The particle velocity into the boundary equals the particle velocity out of the boundary on the other side. The acoustic wave pressure distribution may be developed from transmission line theory. The subscripts 1 and 2 refer to the first and second media.

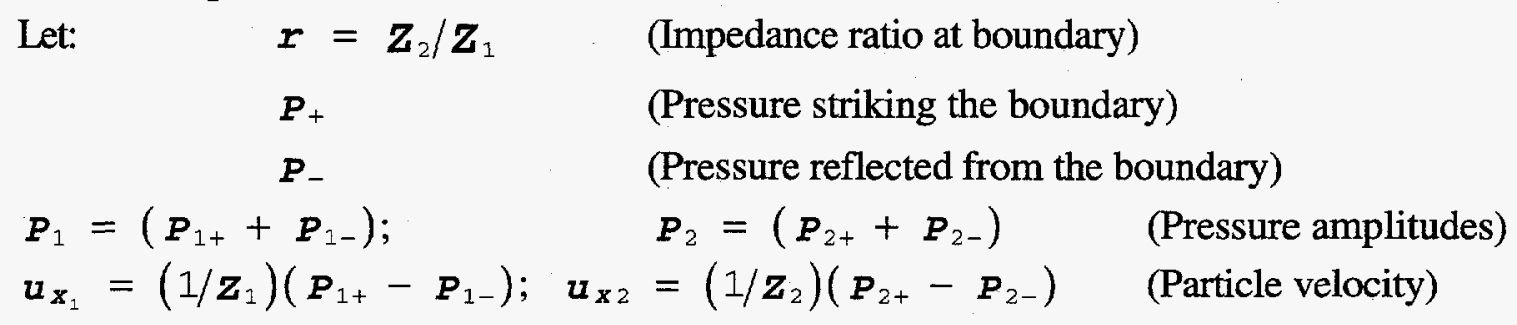

From the assumed boundary conditions,

$$
\begin{aligned}
& \boldsymbol{r} \boldsymbol{P}_{1+}-\boldsymbol{r} \boldsymbol{P}_{1-}=\boldsymbol{P}_{2+} \quad \text { or } \quad \boldsymbol{Z}_{2}\left(\boldsymbol{P}_{1+}-\boldsymbol{P}_{1-}\right)=\boldsymbol{Z}_{1} \boldsymbol{P}_{2+} \\
& \boldsymbol{r} \boldsymbol{P}_{1+}-\boldsymbol{r} \boldsymbol{P}_{1-}=\boldsymbol{P}_{1+}+\boldsymbol{P}_{1-} \\
& \boldsymbol{r} \boldsymbol{P}_{1+}-\boldsymbol{P}_{1+}=\boldsymbol{r} \boldsymbol{P}_{1-}+\boldsymbol{P}_{1-} \\
& \boldsymbol{P}_{1+}(\boldsymbol{r}-1)=\boldsymbol{P}_{1-}(\boldsymbol{r}+1) \\
& \boldsymbol{P}_{1-} / \boldsymbol{P}_{1+}=(\boldsymbol{r}-1) /(\boldsymbol{r}+1) \quad \text { (normal incidence pressure reflectivity) } \\
& \boldsymbol{P}_{2+}=\boldsymbol{P}_{1+}+\boldsymbol{P}_{1-} \quad \text { (pressure partitioning) } \\
& \boldsymbol{P}_{1-}=\boldsymbol{P}_{2+}-\boldsymbol{P}_{1+} \\
& \boldsymbol{Z}_{2}\left(\boldsymbol{P}_{1+}-\boldsymbol{P}_{1-}\right)=\boldsymbol{Z}_{1} \boldsymbol{P}_{2+} \\
& \boldsymbol{Z}_{2}\left(\boldsymbol{P}_{1+}-\left(\boldsymbol{P}_{2+}-\boldsymbol{P}_{1+}\right)\right)=\boldsymbol{Z}_{1} \boldsymbol{P}_{2+} \\
& \boldsymbol{Z}_{2}\left(\boldsymbol{P}_{1+}-\boldsymbol{P}_{2+}+\boldsymbol{P}_{1+}\right)=\boldsymbol{Z}_{1} \boldsymbol{P}_{2+}
\end{aligned}
$$




$$
\begin{gathered}
\boldsymbol{Z}_{2}\left(2 \boldsymbol{P}_{1+}-\boldsymbol{P}_{2+}\right)=\boldsymbol{z}_{1} \boldsymbol{P}_{2+} \\
2 \boldsymbol{z}_{2} \boldsymbol{P}_{1+}-\boldsymbol{Z}_{2} \boldsymbol{P}_{2+}=\boldsymbol{z}_{1} \boldsymbol{P}_{2+} \\
2 \boldsymbol{z}_{2} \boldsymbol{P}_{1+}=\boldsymbol{z}_{1} \boldsymbol{P}_{2+}+\boldsymbol{z}_{2} \boldsymbol{P}_{2+}=\boldsymbol{P}_{2+}\left(\boldsymbol{z}_{1}+\boldsymbol{z}_{2}\right) \\
\boldsymbol{P}_{2+} / \boldsymbol{P}_{1+}=2 \boldsymbol{z}_{2} /\left(\boldsymbol{z}_{2}+\boldsymbol{z}_{1}\right)=2 \boldsymbol{r} /(\boldsymbol{r}+1) ; \quad \text { (normal incidence pressure }
\end{gathered}
$$

transmissivity)

PRESSURE COEFFICIENT $\left(\mathrm{N} / \mathrm{m}^{2}\right)$ :

$$
\begin{array}{rlr}
\boldsymbol{R}_{\boldsymbol{P}}= & (\boldsymbol{r}-1) /(\boldsymbol{r}+1) & \\
\boldsymbol{T}_{\boldsymbol{P}}= & 2 \boldsymbol{r} /(\boldsymbol{r}+1) & \\
\text { Where: } & \boldsymbol{Z}_{2}>\boldsymbol{z}_{1}: \boldsymbol{r}>1 & \\
& \boldsymbol{z}_{2}<\boldsymbol{z}_{1}: \boldsymbol{r}<1 & \text { (In-phase) }
\end{array}
$$

INTENSITY COEFFICIENT $\left(W / \mathrm{m}^{2}\right)$ :

$$
\begin{aligned}
& \boldsymbol{R}_{\boldsymbol{E}}(\boldsymbol{r}-1)^{2} /(\boldsymbol{r}+1)^{2} \\
& \boldsymbol{T}_{\boldsymbol{E}}=4 \boldsymbol{z}_{2} \boldsymbol{z}_{1} /\left(\boldsymbol{z}_{2}+\boldsymbol{z}_{1}\right)^{2}=4 \boldsymbol{r} /(\boldsymbol{r}+1)^{2}
\end{aligned}
$$

The acoustic measurements for the extensional and torsional (shear) wave velocities may be used to calculate the various moduli in homogeneous, isotropic solids. The following Table of Material Properties provides a listing of typical formulae for determining the moduli.

\section{TABLE OF MATERIAL PROPERTIES}

Poisson's Ratio:

Young's Modulus:

Shear Modulus:

Bulk Modulus:

Lame Constant:

Bulk Compressional Velocity:

$$
\begin{aligned}
& v=\left[C_{o}^{2} /\left(2 C_{t}^{2}\right)\right]-1 \\
& \boldsymbol{E}=C_{o}^{2} \rho \\
& G=C_{t}^{2} \rho \\
& K=C_{o}^{2} C_{t}^{2} \rho /\left(3\left(3 C_{t}^{2}-C_{o}^{2}\right)\right) \\
& \lambda=v E /((1+v)(1-2 v)) \\
& C_{L}=C_{t} \sqrt{\left(C_{o}^{2}-4 C_{t}^{2}\right) /\left(C_{o}^{2}-3 C_{t}^{2}\right)}
\end{aligned}
$$

\section{TABLE OF VARIABLES}

A Ultrasonic reflection from the delay line to specimen interface

$\alpha \quad$ Attenuation coefficient, nepers per unit length (nepers $/ \mathrm{m}$ )

B, C First and second ultrasonic reflections from the specimen to air interface

C Acoustic velocity $(\mathrm{m} / \mathrm{s})$

$\mathrm{C}_{\mathrm{t}} \quad$ Torsion wave velocity

D Diameter (meters)

f frequency $(\mathrm{Hz})$

Io Intensity of ultrasound $\left(\mathrm{W} / \mathrm{m}^{2}\right)$

L Length (meters)

$\lambda \quad$ Wavelength, distance per cycle of acoustic waveform ( $\mathrm{m} /$ cycle $)$

no Wavelength of extensional wave 
$\lambda t \quad$ Wavelength of torsional wave

$\mathrm{N} \quad$ An integer value $(1,2,3, \ldots$ etc. $)$

$\mathrm{P}_{+} \quad$ Positive acoustic pressure wave

P. Negative acoustic pressure wave

$\mathrm{P}_{1} \quad$ Acoustic pressure in the first medium

$\mathrm{P}_{2} \quad$ Acoustic pressure in the second medium

$\mathrm{R} \quad$ Ultrasonic reflection coefficient, propagation in direction of transducer to specimen at the time of reflection

$r \quad$ Ratio of the acoustic impedance of the second medium to the acoustic impedance of the first medium

$\mathrm{R}^{\prime} \quad$ Returning ultrasonic reflection coefficient, propagation in direction of specimen to transducer at the time of reflection

$R_{E} \quad$ Intensity reflection coefficient

$R_{P} \quad$ Pressure reflection coefficient

$\mathrm{T}$ Ultrasonic transmission coefficient, propagation in direction of transducer to specimen

$\mathrm{T}^{\prime} \quad$ Returning ultrasonic transmission coefficient, propagation in direction of specimen to transducer

$\mathrm{T}_{\mathrm{E}} \quad$ Intensity transmission coefficient

$\mathrm{T}_{\mathrm{P}} \quad$ Pressure transmission coefficient

$\mathbf{u}_{\mathbf{X} 1} \quad$ Acoustic particle velocity in first medium

$\mathrm{u}_{\mathrm{X} 2}$ Acoustic particle velociy in second medium

$\mathrm{Z}_{1} \quad$ Acoustic impedance of first medium

$\mathrm{Z}_{2} \quad$ Acoustic impedance of second medium

\section{THIN WALL STRUCTURES}

Acoustic and ultrasonic waves may propagate in relatively thin plates, in terms of the wavelength of the propagating sound. The sound waves will propagate as resonances of the structure and may, under some circumstances, be highly dispersive. There will often be either plane harmonic waves or overtones formed within such structures and these waves become the subject of Modal analysis and Acoustic Emission analysis. The following Table suggests some of the modes of sound propagation found in thin wall structures: 
WAVE SOLUTION APPROXIMATIONS 24

\begin{tabular}{|lll|}
\hline \multicolumn{1}{|c}{ Mode } & Displacement & \multicolumn{1}{c|}{$\mathbf{f D}=\begin{array}{c}\text { frequency X thickness, } \\
\text { m }=\text { integer }\end{array}$} \\
\hline \hline Symmetric & Normal & $(2 m-1)\left(C_{S} / \sqrt{2}\right)$ \\
Asymmetric & Normal & $2 m\left(C_{S} / \sqrt{2}\right)$ \\
Symmetric & Normal & $(2 m-1)\left(C_{L} / 2\right)$ \\
Asymmetric & Normal & $2 m\left(C_{L} / 2\right)$ \\
Symmetric & In Plane & $2 m\left(C_{S} / 2\right)$ \\
Asymmetric & In Plane & $(2 m-1)\left(C_{S} / 2\right)$ \\
Symmetric & In Plane & $m C_{L} C_{S} /\left(C_{L}{ }^{2}-C_{S}{ }^{2}\right)^{1 / 2}$ \\
Asymmetric & In Plane & $X_{m} C_{L} C_{S} /\left[\pi\left(C_{L}{ }^{2}-C_{S}{ }^{2}\right)^{1 / 2}\right] *$ \\
\hline
\end{tabular}

${ }^{*}$ Transcendental solution for $\mathrm{X}_{\mathrm{m}}: \operatorname{Tan}_{m}=\frac{\left[2-\left(C_{L} / C_{S}\right)^{2}\right]^{1 / 2}}{1-\left(C_{L} / C_{S}\right)^{2}} X_{m}$ $\tan x=b x$, where $x=\frac{\pi f D}{C_{L}}\left(\frac{C_{L}^{2}}{C_{S}^{2}}-1\right)^{1 / 2}$ and $b=\frac{\left[2-\left(C_{L} / C_{S}\right)^{2}\right]^{2}}{1-\left(C_{L} / C_{S}\right)^{2}}$

Solve for the roots, $x_{m}: f D=\frac{x_{m}}{\pi} \frac{C_{L} C_{S}}{\left(C_{L}^{2}-C_{S}^{2}\right)^{1 / 2}}$

\section{VIBRATION FREQUENCY CALCULATIONS}

\section{Flexural Waves}

Criteria: Width $\gg$ Thickness; Waves are highly dispersive; $C_{f} \propto \sqrt{f}$; the larger the area, the greater the coupling to fluids.

Where:

$$
C_{f}=\left[\frac{Y k^{2}}{\rho\left(1-v^{2}\right)}\right]^{1 / 4} \omega^{1 / 2}=\left[\frac{c_{o}^{2} k^{2}}{\left(1-v^{2}\right)}\right]^{1 / 4} \omega^{1 / 2}
$$

$\mathrm{k}=$ Radius of gyration: Plates: $a / \sqrt{12}$

$\mathrm{a}=$ Thickness

$\mathrm{Y}=$ Young's modulus

$\omega=2 \pi \mathrm{f}$

$v=$ Poisson's Ratio 


$$
\rho=\text { density }
$$

Bars, width $\cong a$

$$
C_{f}=\left[\frac{Y k^{2}}{\rho}\right]^{1 / 4} \omega^{1 / 2}=\left[c_{o}^{2} k^{2}\right]^{1 / 4} \omega^{1 / 2}=\sqrt{\omega c_{o} k}
$$

Rectangular Bar Clamped at One End

$$
\begin{aligned}
& f_{1}=\frac{0.5596}{L^{2}} C_{O} k \quad \text { Where } \mathrm{k}=\text { Radius of gyration } \\
& k=\frac{a}{\sqrt{\mathbf{1 2}}} \text { for rectangular bars, }
\end{aligned}
$$

where $\mathrm{a}=$ Thickness of bar in the direction of vibration.

$$
\begin{aligned}
& k=\frac{a}{2} \quad \text { for round bars, where a = diameter } \\
& f_{2}=6.267 f_{1} \\
& f_{3}=17.55 f_{1} \text { For the second through fourth tone } \\
& f_{4}=34.39 f_{1}
\end{aligned}
$$

Bar, Both Ends Free

$$
f_{1}=\frac{1.133}{L^{2}} \pi C_{o} k \quad \text { flexural vibration mode }
$$

\section{Longitudinal Vibration of Bars}

$$
f_{1}=\frac{C_{O}}{\lambda}=\frac{C_{O}}{2 L}=\frac{1}{2 L} C_{O} \quad \text { Plus Harmonics }
$$

\section{Circular Plate unsupported (Free)}

$$
f_{1}=\frac{0.412 t}{R^{2}} C_{o} \sqrt{\frac{1}{1-v^{2}}} \quad f_{2}=\frac{0.193 t}{R^{2}} C_{o} \sqrt{\frac{1}{1-v^{2}}}
$$

Where: $\mathrm{t}=$ thickness, $\mathrm{R}=$ radius, $v=$ Poisson's Ratio Modes of Vibration

Circular Plate Lightly Supported at the Outside Edge

$$
f_{1}=\frac{0.233 t}{R^{2}} C_{o} \sqrt{\frac{1}{1-v^{2}}}
$$

Circular Plate Clamped at Outside Edge

$$
f_{01}=\frac{0.467 t}{R^{2}} C_{o} \sqrt{\frac{1}{1-v^{2}}}
$$

With Overtones 


$$
\begin{array}{ll}
f_{02}=3.91 f_{01} & f_{03}=8.75 f_{01}
\end{array} \quad f_{11}=2.09 f_{01}
$$

\section{Pipes, Closed One End}

$$
f_{1}=\frac{C}{\lambda}=\frac{C}{4 L}
$$

Where $\mathrm{C}$ is the velocity of sound in the gas within the pipe, and the length is an odd multiple of quarter wavelengths.

\section{Open Pipes}

$$
f_{1}=\frac{C}{\lambda}=\frac{C}{2 L}
$$

Where the length is an even multiple of quarter wavelengths.

\section{CONCLUSIONS}

The specialized techniques ${ }^{15}$ that are available at the Acoustic and Ultrasonic Properties of Materials Laboratory allow us to perform difficult ultrasonic velocity measurements as well as other kinds of acoustic measurement, including acoustic emission detection. We are continuously upgrading techniques through ongoing research projects, and stay abreast of new methods through close contact with researchers around the world. The personnel at the Acoustic Laboratory are always available to discuss and assist with unique problems from requestors.

\section{ACKNOWLEDGMENTS}

The author wishes to express his appreciation to D. R. Green for his valuable experimental contributions, including his discovery of Saran Wrap as an ultrasonic coupling agent, to George Yanes for his assistance in computer programming and electronic instrumentation, and to Dr. C. A. Tatro for his valuable consultation.

\section{REFERENCES}

1. R. Truell, C. Elbaum, and B. B. Chick, Ultrasonic Methods in Solid State Physics (Academic Press, New York, 1969), pp. 53-158, 183-186, 365-368.

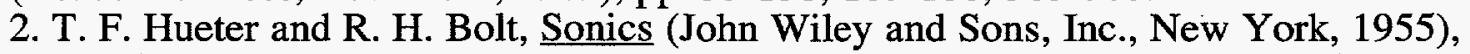
pp. 32-38.

3. H. J. McSkimin, J. Acoust. Soc. Am. 33,(1),12-16 (1961).

4. H. J. McSkimin and P. Andreatch, J. Acoust. Soc. Am. 34, 609-615 (1962).

5. H. J. McSkimin, J. Acoust. Soc. Am. 33,(1), 12-16 (1961).

6. E. P. Papadakis, J. Acoust. Soc. Am. 42, 1045-1051 (1967).

7. E. P. Papadakis, J. Acoust. Soc. Am. 40, 863-876 (1966).

8. J. F. Nye, Physical Properties of Crystals (Oxford University Press, London, 1957). 
9. B. Carlin, Ultrasonics (McGraw-Hill, New York, 1960), pp. 33-36.

10. R. Goldman, Ultrasonic Technology (Reinhold Publishing Corp., New York, 1962).

11. H. L. Dunegan, "High Temperature Dynamic Modulus Measurements by Use of

Ultrasonics," Materials Evaluation 22 (6), 266-272 (1964).

12. H. J. McSkimin, Physical Acoustics, W. P. Mason, Ed. (Academic Press, New York and London, 1966), vol. 1, part A, pp. 272-334.

13. M. B. Reynolds, "The Determination of the Elastic Constants of Metals by the Ultrasonic Pulse Technique," Trans. Am. Soc. for Metals 45, 839-861 (1953).

14. C. F. Cline, H. L. Dunegan, and G. W. Henderson, J. Acoust. Soc. Am. 38(4), 1944-1948 (1967).

15. L. C. Lynnworth, Materials Evaluation, 25(12), 265-277 (1967).

16. L. E. Kinsler and A. R. Frey, Fundamentals of Acoustics (John Wiley \& Sons, New York, 1962), 2nd ed.

17. F. Filipczynski, A. Pawlowski, and J. Wehr, Ultrasonic Methods of Testing Materials (Butterworth \& Co., London, 1966). [Translation].

18. C. F. Brockelsby, J. S. Palfreeman, and R. W. Gibson, Ultrasonic Delay Lines

(London Iliffe, Book Dorset House, Stanford Street, London, 1963).

19. H. J. McSkimin, J. Acoust. Soc. Am. 37(5), 864-871 (1965).

20. H. Seki, A. Granato, and R. Truell, J. Acoust. Soc. Am. 28(2), 230-238 (1956).

21. R. C. McMaster, Ed., Nondestructive Testing Handbook (American Society for

Nondestructive Testing, The Ronald Press Co., New York, 1959), vol. 2, p. 43.11.

22. O. L. Anderson, Physical Acoustics, W. P. Mason, Ed. (Academic Press, New York and London, 1965), vol. 3, part B, pp. 43-95.

23. O. L. Anderson, E. Schreiber, and R. C. Lieberman, Review of Geophysics 6(4), 491-524 (1968).

24. D.M. Egle, A.E. Brown, "Considerations for the Detection of Acoustic Emission

Waves in Thin Plates", J. Acoust. Soc. Am. 57(3), 1975.

25. E. P. Papadakis, J. Acoust. Soc. Am. Vol. 44, No. 5, 1437-1441 (1968).

\section{ABOUT THE REFERENCES}

A larger list of references could undoubtedly have been presented. However, each of these references contains an ample supply of references, should a more extensive investigation be warranted.

The first fifteen references are discussed in the text of this report. Of the remaining eight, the ASNT handbook ${ }^{21}$ contains a most liberal review of techniques, along with some limited theory. This handbook is currently under revision, and when the new edition becomes available, it may contain much of the information on pulse overlap and pulse superposition contained in references $1,3,4,5,6,7,19$, and 20 . References 22 and 23 contain information on the techniques of determining the dynamic elastic moduli in geologic formation, and much of this theory is applicable to a variety of other structural materials. References 16 and 17 contain general ultrasonic theory and information on methods, similar to the information presented in references 2,9 , and 10 . 
Recent advancements in the technology of detecting and propagating ultrasonic waves in filaments and thin wires 15,18 have been of great value to some industries concerned with product quality control, and measurements of elastic constants over extreme temperature ranges. These advancements were made possible by new designs in ultrasonic transducers. Should this technology become required at LLNL, equipment is commercially available for use from $-273^{\circ} \mathrm{C}$ to $5000^{\circ} \mathrm{C}$. 


\section{$\underline{\text { Appendix A }}$}

Mail Station L - 333

Ext: $2-7089$

Current Date

TO : Client

FROM: $\quad$ Al Brown

SUBJECT: $\quad$ DYNAMIC MODULI FOR COMMERCIAL GRADE VANADIUM

The ultrasonic velocities were measured on vanadium plate in preparation for ultrasonic inspection of tubing. The estimated accuracy for the ultrasonic velocities is $\pm 1 \%$. It should be noted that the listed values may be specimen specific.

\begin{tabular}{|lc|}
\hline Specimen I.D. & $\begin{array}{c}\text { Vanadium } \\
\text { Geometry (cm) }\end{array}$ \\
Thickness (mm) & 1.55 \\
Density (kg/m³) & 6,134 \\
Frequency, MHz (VI) & 30.00 \\
Frequency, MHz (Vs) & 20.00 \\
Longitudinal Velocity (m/s) & 5,965 \\
Shear Velocity (m/s) & 2,626 \\
Thin Rod Velocity (m/s) & 4,362 \\
Rayleigh Velocity (m/s) & 2,465 \\
Poisson's Ratio & 0.3798 \\
Young's Modulus (GPa) & 116.7 \\
Shear Modulus (GPa) & 42.3 \\
Lame' Modulus (GPa) & 133.7 \\
Bulk Modulus (GPa) & 161.9 \\
\hline
\end{tabular}

Lower test frequencies were found inadequate. At $30 \mathrm{MHz}$, there are approximately 8 wavelengths of material thickness thereby avoiding the possibility of wave interference. The proposed tubing of the same material has a nominal wall thickness of $0.635 \mathrm{~mm}$ ( 0.025 inch), about 3 wavelengths thick at $30 \mathrm{MHz}$. A $30 \mathrm{MHz}$, highly damped broad band transducer will probably suffice if used with a wide bandwidth, high frequency receiver. A crack width of $50.8 \mu \mathrm{m}(0.002 \mathrm{inch})$ will be approximately one quarter wavelength and will reflect the sound at $30 \mathrm{MHz}$ if the crack length is in-plane and is at least 3.5 wavelengths $(0.8 \mathrm{~mm})$ long oriented normal to the sound propagation direction. A $30 \mathrm{MHz}$ transducer with a spherical focal length of one inch should be able to detect a flaw of $8 \mathrm{~nm}^{2}\left(13 \mu \mathrm{in}^{2}\right)$ area. 


\section{Appendix B}

\section{ATTENUATION OF ULTRASOUND}

\section{GENERAL EQUATIONS:}

For pulse-echo

$$
\frac{I_{2}}{I_{1}}=e^{-2 \alpha} ; \frac{P_{2}}{P_{1}}=e^{-\alpha} ; \frac{d B}{\lambda}=\frac{d B}{\mu s} * \frac{10^{6}}{\text { freq }} ; \frac{d B}{m}=\frac{d B}{\mu s} * \frac{10^{6}}{\text { velocity }}
$$

Where:

$I_{2} / I_{1}=$ ratio of intensities at two points a unit distance apart, and $I_{2}<I_{1}$.

$\mathrm{P}_{2} / \mathrm{P}_{1}=$ ratio of pressures at two points a unit distance apart, and $\mathrm{P}_{2}<\mathrm{P}_{1}$.

$\alpha=$ attenuation constant (nepers/unit distance)

$\alpha^{\prime}=8.686 \alpha(\mathrm{dB} / \mathrm{cm})$

$\mathrm{A}=\lambda \alpha$ (nepers/wavelength) where $\lambda$ equals velocity/frequency

$\mathrm{A}_{1}=8.686 \lambda \alpha(\mathrm{dB} /$ wavelength $)$

\section{ATTENUATION RESULTS FROM:}

1) Beam spread

(finite transducer size)

2) Field effects

3) Couplant mismatch

(direct couplant reflection loss, waterbuffer)

4) Transducer loading (couplant or specimen)

5) Sample geometry

(defect reflection: size, shape, surface, impedance)

6) Diffraction pattern

(losses from beam divergence, wave front phase effects)

7) Non parallelism

(rough surfaces)

8) Absorption:

a) Dislocation damping (radiation damage, internal oxidation)

b) Motion of domain walls

c) Magnetic "rotation" effect near saturation

d) Visco-elastic losses (thermo-elastic losses, diffusion of atoms) 
9) Scattering:

a) By small objects

b) By extended (internal) surfaces (reflection)

c) By wave front phase effects (bending and distortion) due to space variation, velocity, refraction index

10) Diffusion

11) Viscous damping losses

12) Relaxation losses

And, finally, attenuation caused by grain size, yield strength, crystalline damage, impact strength, and hardness. 


\section{Appendix C}

\section{ATTENUATION MEASUREMENTS25}

\section{BUFFER ROD TECHNIQUE}

The reflection coefficient at the buffer-sample interface must have a moderate value so that the transmitted and reflected portions of the wave at the buffer-sample interface have comparable amplitudes. Nearly equal acoustic impedances in the two materials are required.

The loss per echo of the multiple echoes in the buffer rod must be sufficient in the short buffer case to ensure that these echoes do not interfere with the echoes returning from the sample.

In the long buffer rod, the attenuation should be very low. In either case, the sample echoes should not coincide with the buffer echoes, but have well-separated echoes.

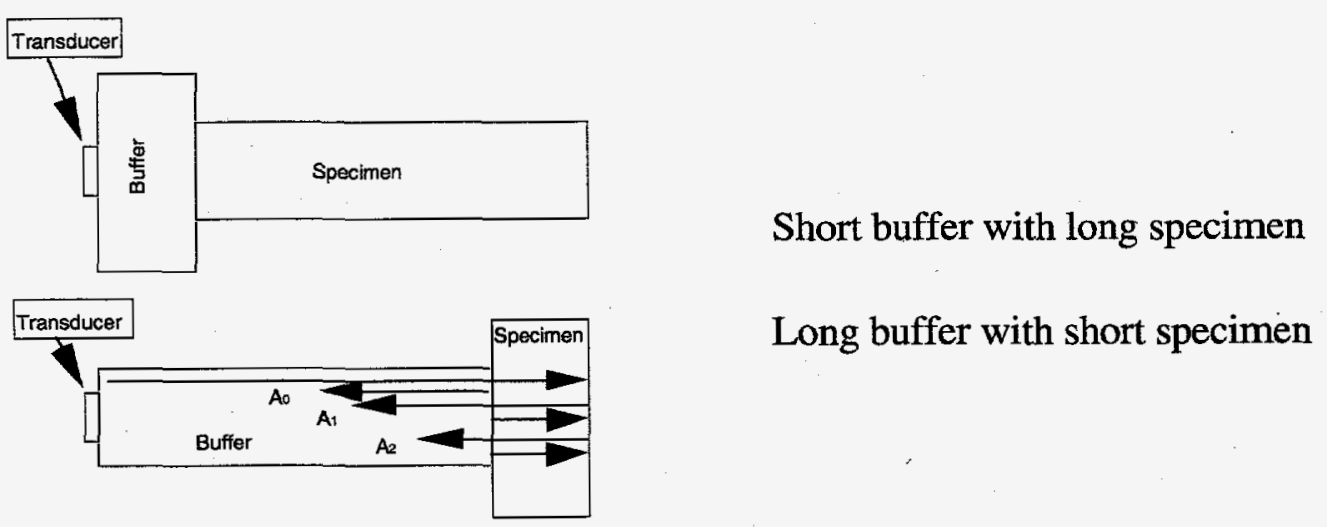

\section{FORWARD PROPAGATION}

$R=\frac{Z_{2}-Z_{1}}{Z_{2}+Z_{1}} \quad T=R+1=\frac{Z_{2}-Z_{1}}{Z_{2}+Z_{1}}+1$

REVERSE PROPAGATION

$R^{\prime}=\frac{Z_{1}-Z_{2}}{Z_{1}+Z_{2}} \quad T^{\prime}=\frac{Z_{1}-Z_{2}}{Z_{1}+Z_{2}}+1$

DEFINITIONS 
$A_{0}=R=\frac{Z_{2}-Z_{1}}{Z_{2}+Z_{1}} \quad$ First Interface Reflection, Buffer-Specimen

$A_{1}=T T^{\prime}=\left(\frac{Z_{2}-Z_{1}}{Z_{2}+Z_{1}}+1\right)\left(\frac{Z_{1}-Z_{2}}{Z_{1}+Z_{2}}+1\right)$

$A_{1}=\frac{4 Z_{2} Z_{1}}{\left(Z_{1}+Z_{2}\right)^{2}} \quad$ First Reflection from Specimen Back Surface

But: $A_{1} \propto R \quad$ and $\quad T=R+1 \quad T^{\prime}=1-R$

Therefore: $\quad A_{1}=T T^{\prime}=(R+1)(1-R)=1-R^{2}$

And: $\quad A_{2}=T R^{\prime} T^{\prime}=(R+1)(1-R)(\bar{R})=\bar{R}\left(1-R^{2}\right)$

Where $\bar{R}$ is the phase reversal on the second reflection: numerically the same as $R$, but reversed polarity.

\section{ATTENUATION}

$A_{1}=\left(1-R^{2}\right) e^{-\alpha 2 L}$

$A_{2}=\bar{R}\left(1-R^{2}\right) e^{-\alpha 4 L}$

$\alpha=$ nepers per unit length

\section{AMPLITUDE RATIO}

$B_{0}=A_{0} / A_{1}=R e^{\alpha 2 L} /\left(1-R^{2}\right)$

$B_{2}=A_{2} / A_{1}=\bar{R} e^{-\alpha 2 L}$

$B_{0} B_{2}=\frac{\left(R e^{\alpha 2 L}\right) \bar{R} e^{-\alpha 2 L}}{1-R^{2}}=\frac{(1) R^{2}}{1-R^{2}}$

$R=\left[B_{0} B_{2} /\left(1+B_{0} B_{2}\right)\right]^{1 / 2}$

$R=\left[\frac{A_{2}}{A_{1}} \frac{A_{0}}{A_{1}} /\left(1+\frac{A_{2}}{A_{1}} \frac{A_{0}}{A_{1}}\right)\right]^{1 / 2}$

$\alpha=\left[\ln \left(R / B_{2}\right)\right] / 2 L$

$\alpha=\left[\ln \left(\left(\frac{A_{2}}{A_{1}} \frac{A_{0}}{A_{1}} /\left(1+\frac{A_{2}}{A_{1}} \frac{A_{0}}{A_{1}}\right)\right)^{1 / 2} / \frac{A_{2}}{A_{1}}\right)\right] / 2 L$ 

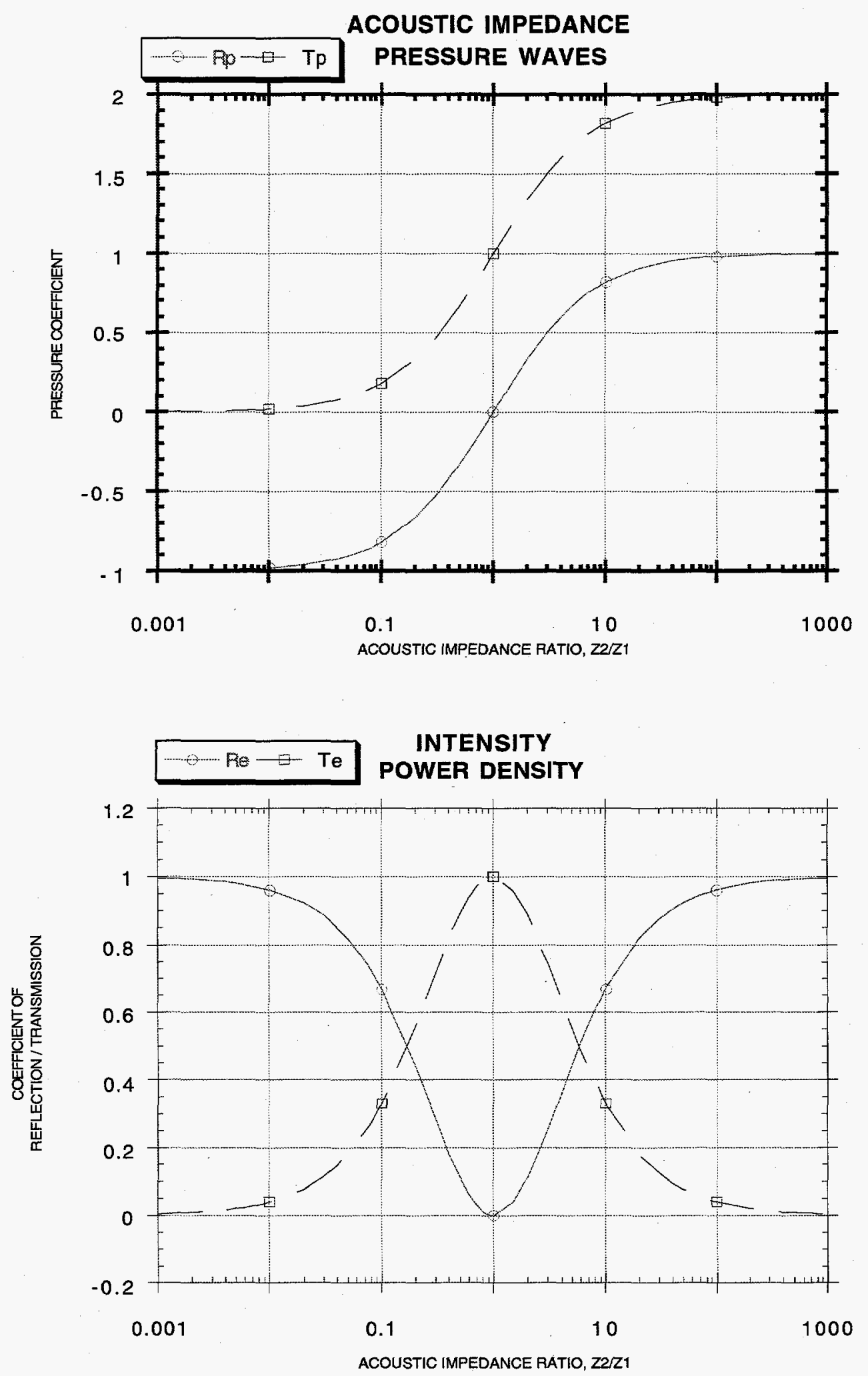